\title{
Tunable Lowpass Filter with RF MEMS Capacitance and Transmission Line
}

\author{
Shimul C. Saha, ${ }^{1,2}$ Ulrik Hanke, ${ }^{3}$ Håkon Sagberg, ${ }^{4}$ Tor A. Fjeldly, ${ }^{1}$ and Trond Sæther ${ }^{1}$ \\ ${ }^{1}$ Department of Electronics and Telecommunications, Norwegian University of Science and Technology, \\ 7491 Trondheim, Norway \\ ${ }^{2}$ Electronics and Nanoscale Engineering Research Division, University of Glasgow, Glasgow G12 8QQ, UK \\ ${ }^{3}$ Institute for Micro and Nano Systems Technology, Vestfold University College, 3103 Tønsberg, Norway \\ ${ }^{4}$ Department of Microsystems and Nanotechnology, SINTEF ICT, P.O. Box 124, Blindern, 0314 Oslo, Norway
}

Correspondence should be addressed to Shimul C. Saha, shimul.saha@iet.ntnu.no

Received 27 June 2011; Accepted 5 October 2011

Academic Editor: Jiun Wei Horng

Copyright (C) 2012 Shimul C. Saha et al. This is an open access article distributed under the Creative Commons Attribution License, which permits unrestricted use, distribution, and reproduction in any medium, provided the original work is properly cited.

\begin{abstract}
We have presented an RF MEMS tuneable lowpass filter. Both distributed transmission lines and RF MEMS capacitances were used to replace the lumped elements. The use of RF MEMS capacitances gives the flexibility of tuning the cutoff frequency of the lowpass filter. We have designed a low-pass filter at 9-12 GHz cutoff frequency using the theory of stepped impedance transmission lines. A prototype of the filter has been fabricated using parallel plate capacitances. The variable shunt capacitances are formed by a combination of a number of parallel plate RF MEMS capacitances. The cutoff frequency is tuned from $\mathrm{C}$ to X band by actuating different combinations of parallel capacitive bridges. The measurement results agree well with the simulation result.
\end{abstract}

\section{Introduction}

Radio frequency microelectromechanical systems (RF MEMSs) technology offers an attractive capability for RF systems, particularly in support of switching and tuning functions. One such component is a microelectromechanical voltage tuneable capacitor, which can enable a wide tuning range and high-quality $(\mathrm{Q})$ factor. In this paper, we present a design of a tuneable low-pass filter combining RF MEMS capacitors and high impedance transmission lines. The low-pass filter has been designed by means of the theory of stepped-impedance transmission line filters. The filter can be developed with surface micromachining without the need for any external inductors. This will give flexibility in integration and easy fabrication processing.

Compared with solid-state varactors, MEMS tuneable capacitors have the advantage of lower loss and a potentially larger tuning range [1]. The interconnection loss and noise can also be reduced compared with the use of off-chip solid-state RF components. Among all the MEMS capacitors developed to date, the parallel plate configuration is the most commonly used. The actuation mechanism is mostly electrostatic as it consumes very little power during actuation. In standard filter design at low frequency, the MEMS varactor is used as a lumped element with off-chip inductors. At high frequency, some low pass filter designs were reported with integrated transmission lines and MEMS switches (see Cai et al. [2] and Fang et al. [3]). In [2] the tuning of the filter is obtained by changing the transmission line length using MEMS switches. In [3] planar spiral inductor and shunt capacitor are used but without any tuning capability. Also, low-pass and band-pass filters integrating capacitive MEMS switches and short inductive transmission lines have been realised above $10 \mathrm{GHz}$ [4-6]. In [4], the tuning of the cutoff frequency was obtained by switching between different combination of inductance and capacitance. In $[5,6]$, the cut-off frequency was tuned by changing the inductance using MEMS switching.

In this paper, we have shown a design of a low-pass filter with a high-impedance transmission line and RF MEMS capacitors, where the tuning is obtained by capacitance tuning. The MEMS switches were designed in shunt configuration on a coplanar wave guide (CPW). The MEMS capacitive shunt switch was used in a stepped-impedance low-pass filter instead of a low-impedance transmission line. The series inductor in the low-pass filter was implemented 
using a short section of a high impedance transmission line [4]. A preliminary theory of low-pass filters using transmission lines and MEMS capacitances is presented in [7, $8]$. This filter is much more elegant and compact than filters based on traditional $\lambda / 8$ long stub and transmission line filters [9]. Capacitance tuning is much more efficient than the inductance and transmission line tuning. To change the inductance and transmission line, additional MEMS switch is required, which increases the complexity and loss in the systems [2-6]. The designed filter has a large tuning range, over $60 \%$ of its nominal cut-off frequency. Although step impedance filters do not have a very sharp rolloff, they can be used for certain applications where compact size and a wide tuning range are required and less rolloff can be accepted. The low-pass filter, for example, can be used in harmonic suppression above the fundamental frequency in frequency synthesizer. The high tuning range will be very useful in the systems with multiple frequency band operation.

The theory of MEMS capacitor and third-order $3 \mathrm{~dB}$ ripple chebyshev low-pass filter is described in Section 2. The filter was designed and optimised with advanced design system (ADS) from Agilent [10]. A prototype of the filter was fabricated by our own facility at SINTEF MiNaLab in Oslo, Norway. Three parallel plate bridges were used as tuneable capacitances. The tuning of the capacitance was obtained by actuating different combinations of bridges. The fabrication of the filter is presented in Section 3. The measurement of the filter is presented in Section 4. A comparison of the measurement result with simulation result of the filter, taking into account the effects of process constraints, has been also discussed in Section 4. Finally, the paper is concluded.

\section{Theory of Capacitive Switch and Third-Order Filter}

2.1. Theory of Parallel Plate Capacitive Switch. An MEMS capacitive switch consisting of a suspended top plate and a fixed bottom electrode plate with an overlap area $A_{e}=$ $L_{e} \times W_{e}$ is shown in Figure 1. $L_{e}$ and $W_{e}$ are the length and the width of the electrode, respectively. A DC bias voltage, $V_{\mathrm{DC}}$, is applied between the bridge and the two separate actuation electrodes on the two sides of the centre conductor as shown in Figure 1. All electrodes are covered with a dielectric with thickness $t_{d}$ and relative permittivity $\varepsilon_{r}$. The initial gap is $g_{0}$ at the centre of middle electrode when no DC voltage is applied. When a DC voltage is applied between the bridge and the actuation electrodes, the initial gap reduces according to the actuation voltage [11]. If the fringing field effect is neglected, the value of the capacitance between the suspended bridge and centre conductor for a deflection $x$ of the bridge can be determined by

$$
C(x)=\varepsilon_{0} \cdot \frac{A_{e}}{\left(g_{0}-x\right)+t_{d} / \varepsilon_{r}} .
$$

When $V_{\mathrm{DC}}$ is applied between actuation electrodes and bridge, an attractive electrostatic force $F_{e}$ is generated with the value:

$$
F_{e}=\frac{1}{2} \frac{C_{a}(x) V_{\mathrm{DC}}{ }^{2}}{\left(g_{a 0}-x+t_{d} / \varepsilon_{r}\right)} .
$$

Here, $C_{a}(x)$ is the total capacitance between the bridge and the two actuation electrodes, $x$ is the distance the top plate has moved from its initial position, $V_{\mathrm{DC}}$ is the actuation voltage, and $g_{\text {ao }}$ is the initial gap between actuation electrodes and suspended bridge. This electrostatic force tries to pull the top plate down. The beam has a mechanical spring constant (stiffness) which tries to pull the beam back to the upstate position. For small deflections, the electrostatic and elastic force are equal in magnitude. When the beam is deflected one-third of the initial gap, the electrostatic force becomes stronger than the mechanical restoring force. At this point the beam collapses fully to the down state and this voltage is called the pull-down voltage. The down state capacitance is given by

$$
C_{d}=\varepsilon_{0} \varepsilon_{r} \frac{A_{e}}{t_{d}}
$$

2.2. Theory of the Stepped-Impedance Filter. It is well known [9] that if the length of the transmission line is short $(\beta l<\pi / 4)$ and the characteristic impedance is high, the transmission line can be represented by the equivalent circuit shown in Figure 2(a) where the reactance is given by

$$
X_{L}=Z_{0} \beta \ell
$$

Here, the characteristic impedance of the line is $Z_{0}$, the length is $l$, and the propagation constant is $\beta$. So, a series inductor can be represented by a short length of high-characteristic-impedance transmission line. In the lumped elements filter model, the shunt capacitors have been replaced by an RF MEMS capacitor which will provide the capability of tuning cut-off frequency.

2.3. A Third-Order Lowpass Filter Topology Design. A thirdorder low pass filter topology is shown in Figure 2(b). The basic filter elements are extracted from the Chebyshev equal ripple low pass filter prototype [7-9]. The normalized filter elements for a $3 \mathrm{~dB}$ ripple Chebyshev are given by $g_{1}=3.3487, g_{2}=0.7117, g_{3}=3.3487$, and $g_{4}=$ 1.00 which is the load impedance [9]. A ripple of $3 \mathrm{~dB}$ is used to give a sharper roll-off above the cut-off frequency. We have chosen a nominal cut-off frequency at $7.0 \mathrm{GHz}$. With a maximum transmission line impedance of $80 \Omega$, the length of the transmission line becomes $25.5^{\circ}$ and the nominal capacitance value becomes $1.52 \mathrm{pF}$ when the cutoff frequency is $7 \mathrm{GHz}$. The proposed filter is fabricated on a $280 \mu \mathrm{m}$ think high-resistivity silicon substrate. The relative dielectric constant of the substrate is 11.9 and the resistivity is $8000 \Omega$-cm. After optimization of the filter cut-off frequency in ADS, the nominal shunt capacitor value becomes $1.1 \mathrm{pF}$ and the length of the $80 \Omega$ line becomes $1467 \mu \mathrm{m}\left(30.7^{\circ}\right)$ for the desired responses [10]. For a $50 \Omega \mathrm{CPW}$ transmission line, the dimensions are $80 \mu \mathrm{m} / 140 \mu \mathrm{m} / 80 \mu \mathrm{m}\left(G / W_{c} / G\right)$. 


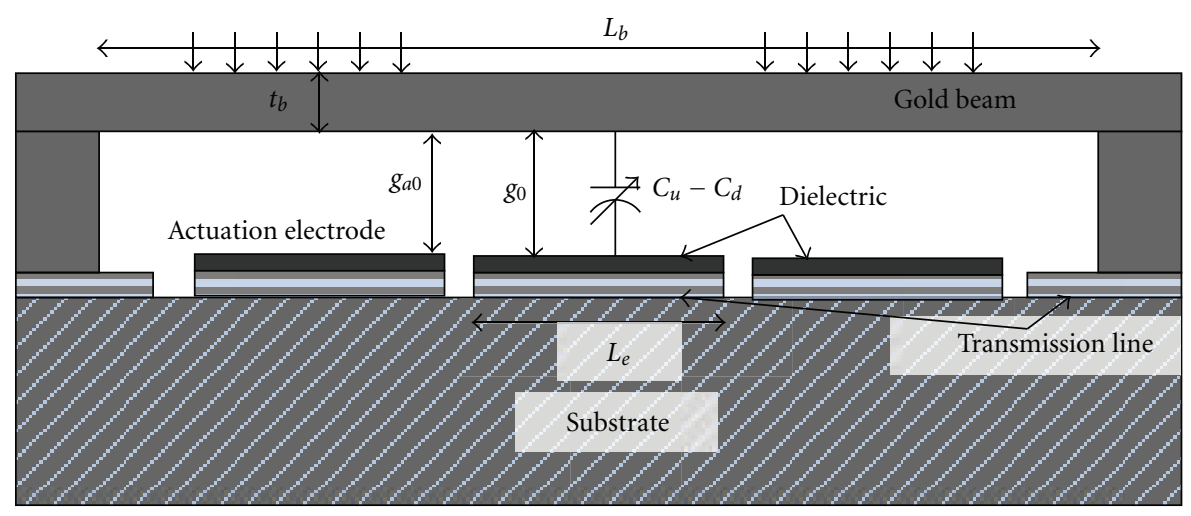

FIGURE 1: Side view of a traditional parallel plate capacitive switch in CPW configuration with separate actuation electrodes.

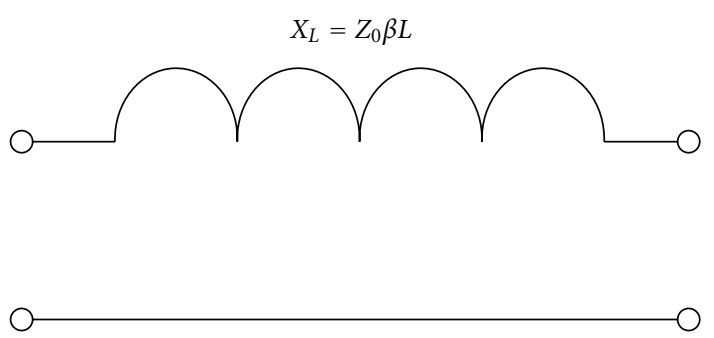

(a)

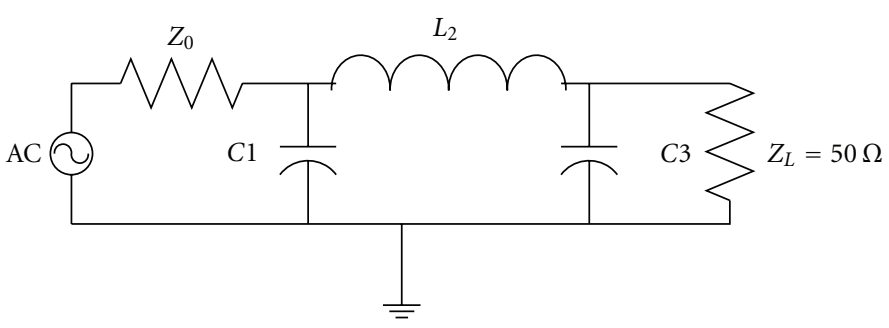

(b)

FIGURE 2: (a) Equivalent circuit for short length and high characteristic impedance transmission line and (b) third order low-pass filter topology.

For an $80 \Omega \mathrm{CPW}$ transmission line, the dimensions are $160 \mu \mathrm{m} / 53 \mu \mathrm{m} / 160 \mu \mathrm{m}(G / W / G)$. A higher value, such as $100 \Omega$ or more, can be chosen for the impedance of the series inductance. This will make the transmission line very narrow and increase the loss in pass band.

\section{Fabrication of the Filter}

A schematic view of the fabricated filter is shown in Figure 3. The fabrication was performed at SINTEF MiNaLab in Oslo. Each shunt capacitance was a combination of two $12 \mu \mathrm{m}$ wide (on the two sides) and one $15 \mu \mathrm{m}$ wide (middle) shunt bridge. The variation of the capacitance can be obtained by actuating individual bridges, or combining one or more bridges. The thickness of the centre electrode and the actuation electrode dielectric was $\left(\mathrm{Si}_{3} \mathrm{~N}_{4}\right) 220 \mathrm{~nm}$. Aluminium was used as a transmission line.

A four-mask process was used to fabricate the proposed filter. A $280 \mu \mathrm{m}$ thick silicon wafer with $4-8 \mathrm{k} \Omega-\mathrm{cm}$ (specified by supplier) resistivity was used for the fabrication. A $500 \mathrm{~nm}$ thick oxide was grown on the silicon wafer to obtain lower loss. Then a $500 \mathrm{~nm}$ thick Al was deposited by sputtering and patterned by wet etching. A $220 \mathrm{~nm}$ thick $\mathrm{Si}_{3} \mathrm{~N}_{4}$ was deposited as dielectric by plasma-enhanced chemical vapour deposition (PECVD) and patterned by dry etch. HiPR 6517 photoresist was used as a sacrificial layer. The resist thickness was $2.55 \mu \mathrm{m}$ and patterned by standard lithography and special baking methods as described in detail in [12]. Then, $1.1 \mu \mathrm{m}$ thick gold was sputtered and patterned by wet etch. The gold was sputtered at 500 watt and a pressure of 20 mTorr to obtain a low tensile stress [13]. When the gold was sputtered, the transmission line was also sputtered with $1.1 \mu \mathrm{m}$ additional gold on aluminum. Low tensile stress ensures low-pull-down voltage and gives better reliability. Finally, the shunt capacitive bridges were released by dissolving the resist in acetone and dried in a critical point dryer [12]. Scanning electron microscope (SEM) images of the fabricated filter are shown in Figure 4. As shown in Figure 4, the actuation electrodes are separated from the RF signal electrode, and each bridge has its own set of actuation electrodes. The centre narrow line is the $80 \mathrm{Ohm}$ transmission line, which is equivalent to a series inductor. The capacitors are at both ends of the $80 \mathrm{Ohm}$ transmission line, shunting a $50 \mathrm{Ohm}$ transmission line. In the figure, the three parallel bridges of shunt capacitors can be seen. The extended $50 \mathrm{Ohm}$ transmission lines are used for measurement purpose with a CPW probe.

\section{Measured Results and Simulation in the Light of Fabrication Constraint}

The fabricated filters were measured in a vector network analyser. The filter characteristic was measured up to $30 \mathrm{GHz}$. Standard line-reflect-reflect-match (LRRM) methods were used for calibration of the network analyser. The measured filter $S$ parameters are shown in Figure 5. We measured 


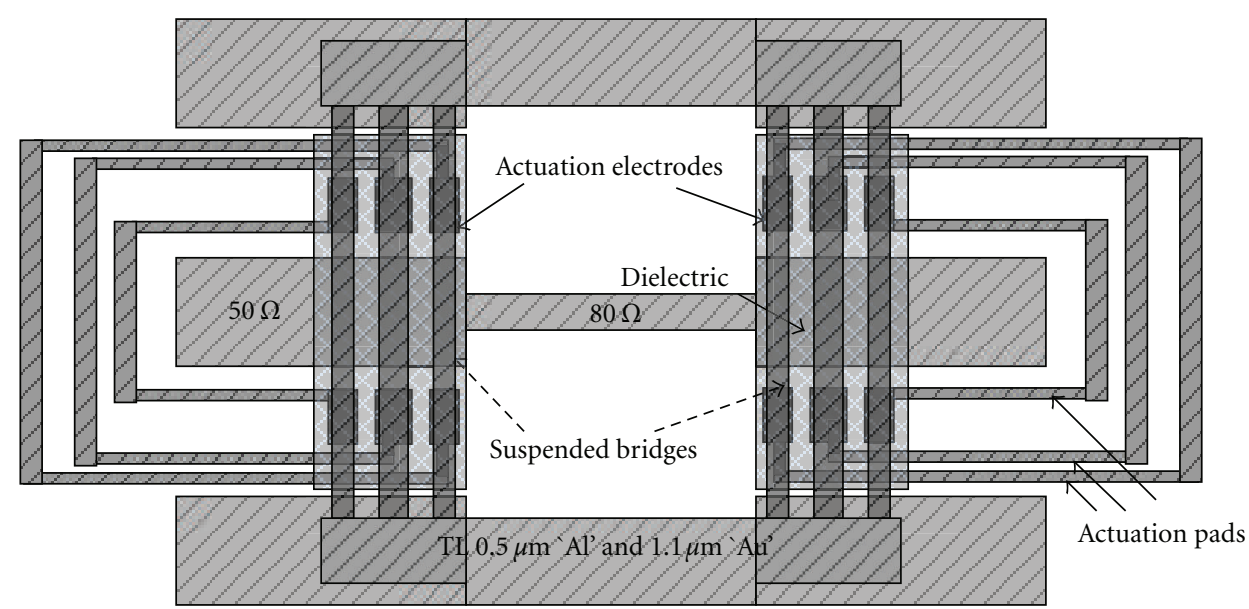

FIgURE 3: The top view of the proposed low pass filter with actuation mechanism.

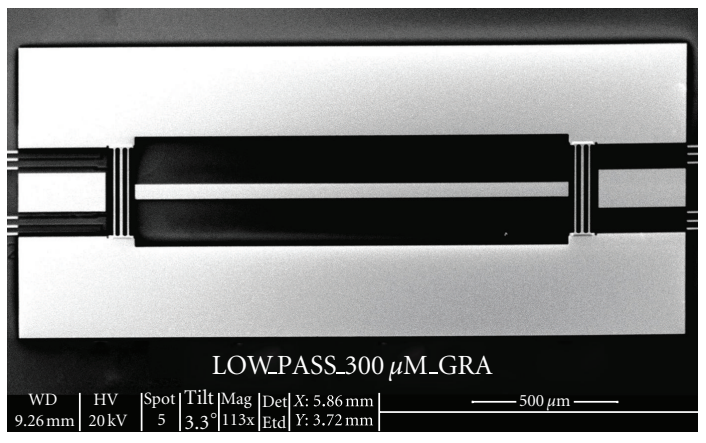

(a)

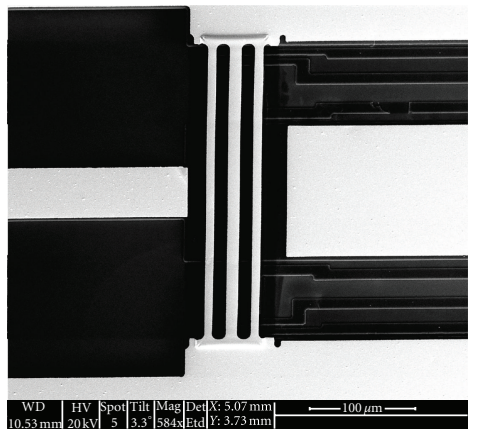

(b)

Figure 4: (a) A SEM image of the fabricated low-pass filter (top view). Three narrow bridges at both sides represent the tunable capacitance. Chip size $(2.3 \mathrm{~mm} \times 1 \mathrm{~mm})$ and (b) an enlarged image of the low-pass filter at the capacitance area.

the filter characteristics with one bridge down $(15 \mu \mathrm{m}$ wide centre bridge), and 2 bridges down $(15 \mu \mathrm{m}$ and $12 \mu \mathrm{m}$ wide bridges). Due to lack of actuation facility, we could not actuate all three parallel bridges together. The cutoff frequency for one bridge down is $12 \mathrm{GHz}$, and two bridges down $9 \mathrm{GHz}$. The filter is very compact compared to the traditional half-wave-length transmission line filter. In addition, the loss in the pass band is reasonably small.

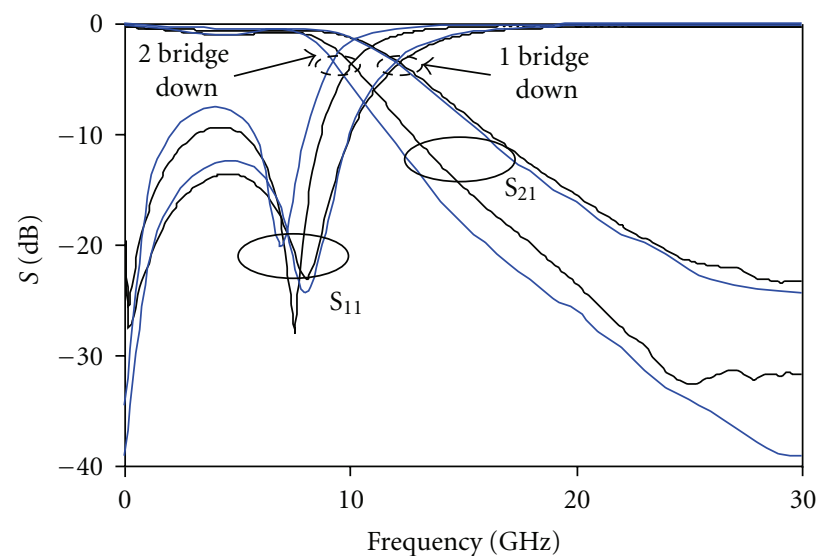

FIGURE 5: Comparison of the filter performances between simulated (blue line) and measured results (black line).

The cut-off frequency was slightly higher than designed frequency. After fabrication, the filters were inspected in an optical interferometer to measure the actual width and height of the bridge. The actual initial heights of the bridges were $2.25 \mu \mathrm{m}$ and the width was reduced on average $1 \mu \mathrm{m}$ from each side owing to overetching. Gold is usually smooth and it was found that the average roughness of the gold was $7 \mathrm{~nm}$. The $\mathrm{Si}_{3} \mathrm{~N}_{4}$ average roughness was $9 \mathrm{~nm}$, but the main contribution to the roughness was from aluminium. This is because of the high-temperature $\left(300^{\circ} \mathrm{C}\right) \mathrm{Si}_{3} \mathrm{~N}_{4}$ deposition. From an atomic force microscope (AFM) measurement, it was found that the height of the aluminium bumps was about $80 \mathrm{~nm}$. As the nitride was deposited on aluminium, the total roughness for the downstate capacitance was $96 \mathrm{~nm}$. Considering the overetch and roughness, the downstate capacitance value is $0.38 \mathrm{pF}$ for the $15 \mu \mathrm{m}$ bridge $(13 \mu \mathrm{m}$ real width). The filter was resimulated with the above parameters. The simulation was compared with measurement results of the fabricated filter as shown in Figure 5. A higher resolution of the measured $S$ parameters is also shown in Figure 6. The measured insertion loss is less than $1.25 \mathrm{~dB}$ in the 


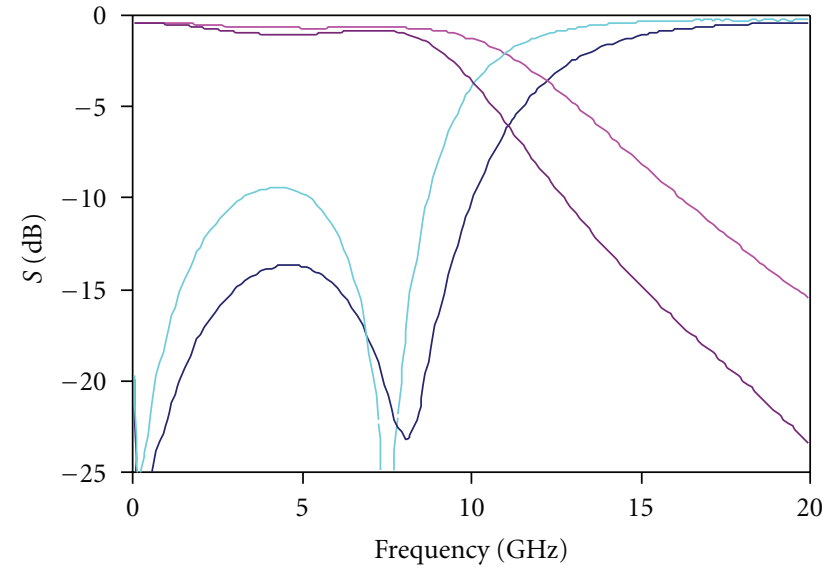

FIGURE 6: The measured filter performances at higher resolution in scale.

pass band for both configurations. The filter has a small amount of ripple in the pass band. The measured results agree well with the simulated results, given the effect of overetching and roughness. There is a slightly higher cut-off frequency in the measured filter. This can happen because of nonplanarity of the bridge at downstate, which will further reduce the downstate capacitance. By using the same roughness as mentioned earlier, the estimated downstate capacitance becomes $0.30 \mathrm{pF}$ for the $12 \mu \mathrm{m}$ bridge $(10 \mu \mathrm{m}$ real width). The total capacitance at downstate becomes $0.68 \mathrm{pF}$ when two bridges are down. The simulated and measured results for this state were compared and are shown in Figure 5. From this figure, it can be seen that the measured filter again has a higher cut-off frequency for the reason discussed above. Also, the narrower bridge will have relatively more overetching and less planarity.

\section{Conclusions}

We have presented a tuneable low-pass filter design with transmission line and MEMS capacitance. The tuning of the cut-off frequency was obtained by actuation of several parallel bridges. The measured results agree well with the simulated result considering the fabrication effects. The filter rolloff is not very sharp but can be improved with the use of high-impedance transmission line and a higher-order of the filter. However, this may have some drawbacks. As only the capacitance was used for tuning and the inductance was fixed, higher order filters would have reduce the tuning range. Also, it would be complicated to tune all the capacitor simultaneously. The fabricated filter is very compact and has very good control of the tuning frequency.

\section{Acknowledgments}

The authors are grateful to the Norwegian Research Council for sponsoring the work through the SMiDA project (no. 159559/130) and the IRRFT Project (no. 159259/I40). The authors also thank MiNa-Lab personnel for their support in the clean room.

\section{References}

[1] J. Zou, C. Liu, J. Schutt-Aine, J. Chen, and S. M. Kang, "Development of a wide tuning range MEMS tunable capacitor for wireless communication systems," in Proceedings of the International Electron Devices Meeting, Technical Digest (IEDM '00), pp. 403-406, December 2000.

[2] M. Cai, X. L. Guo, Y. Li, L. Liu, and L. Zong-sheng, "Design and modeling of reconfigurable MEMS low pass filter," in Proceedings of the 8th International Conference on Solid-State and Integrated Circuit Technology (ICSICT '06), pp. 563-565, October 2006.

[3] J. Fang, Z. W. Liu, Z. M. Chen, L. T. Liu, and Z. J. Li, "Realization of an Integrated Planar LC low-pass filter with Modified Surface Micromachining technology," in Proceedings of the IEEE Conference on Electron Devices and Solid-State Circuits (EDSSC '05), pp. 729-731, December 2005.

[4] D. Peroulis, S. Pacheco, K. Sarabandi, and L. P. B. Katehi, “Tunable lumped components with applications to reconfigurable MEMS filters," in International Microwave Symposium Digest (IEEE-MTT-S '01), vol. 1, pp. 341-344, May 2001.

[5] S. Lee, J. M. Kim, J. M. Kim, Y. K. Kim, and Y. W. Kwon, "Millimeter-wave MEMS tunable low pass filter with reconfigurable series inductors and capacitive shunt switches," IEEE Microwave and Wireless Components Letters, vol. 15, no. 10, pp. 691-693, 2005.

[6] J. H. Park, S. Lee, J. M. Kim, H. T. Kim, Y. Kwon, and Y. K. Kim, "Reconfigurable millimeter-wave filters using CPWbased periodic structures with novel multiple-contact MEMS switches," Journal of Microelectromechanical Systems, vol. 14, no. 3, pp. 456-463, 2005.

[7] S. C. Saha and T. Sæther, "Modeling and Simulation of Low Pass Filter using RF MEMS Capacitance and Transmission line," in Proceedings of the International Microelectronics And Packaging Society (IMPS '05), pp. 155-159, 2005.

[8] S. C. Saha, U. Hanke, and T. Sæther, "Modeling, design and simulation of tunable band pass filter using RF MEMS capacitance and transmission line," in Microelectronics: Design, Technology, and Packaging II, vol. 6035 of Proceedings of SPIE, Brisbane, Australia, December 2005.

[9] D. M. Pozar, Microwave Engineering, chapter 8, John Wiley \& Sons, New York, NY, USA, 2nd edition, 1998.

[10] Advance Design System, from Agilent.

[11] G. M. Rebeiz, RF MEMS Theory, Design and Technology, chapter 2, John Wiley \& Sons, New York, NY, USA, 2nd edition, 2003.

[12] S. C. Saha, H. Sagberg, E. Poppe, G. U. Jensen, T. A. Fjeldly, and T. Sæther, "Tuning of resist slope with hard-baking parameters and release methods of extra hard photoresist for RF MEMS switches," Sensors and Actuators, A, vol. 143, no. 2, pp. 452461, 2008.

[13] S. C. Saha, H. Sagberg, E. Poppe, G. U. Jensen, and T. Sæther, "Metallization scheme and release methods for fabrication of RF MEMS switches," in Proceedings of 33rd Micro- and NanoEngineering conference (MNE'07), 2007. 

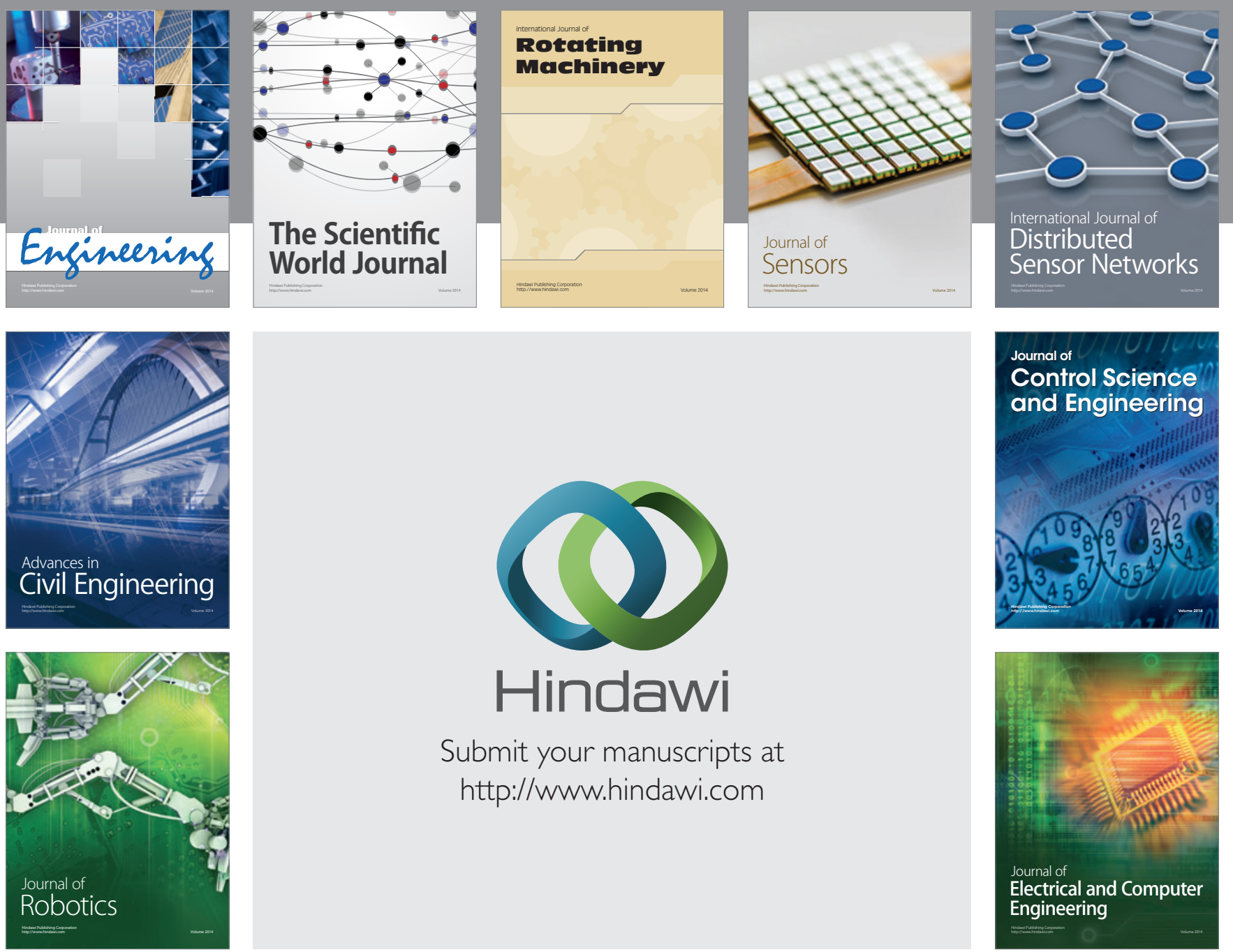

Submit your manuscripts at

http://www.hindawi.com
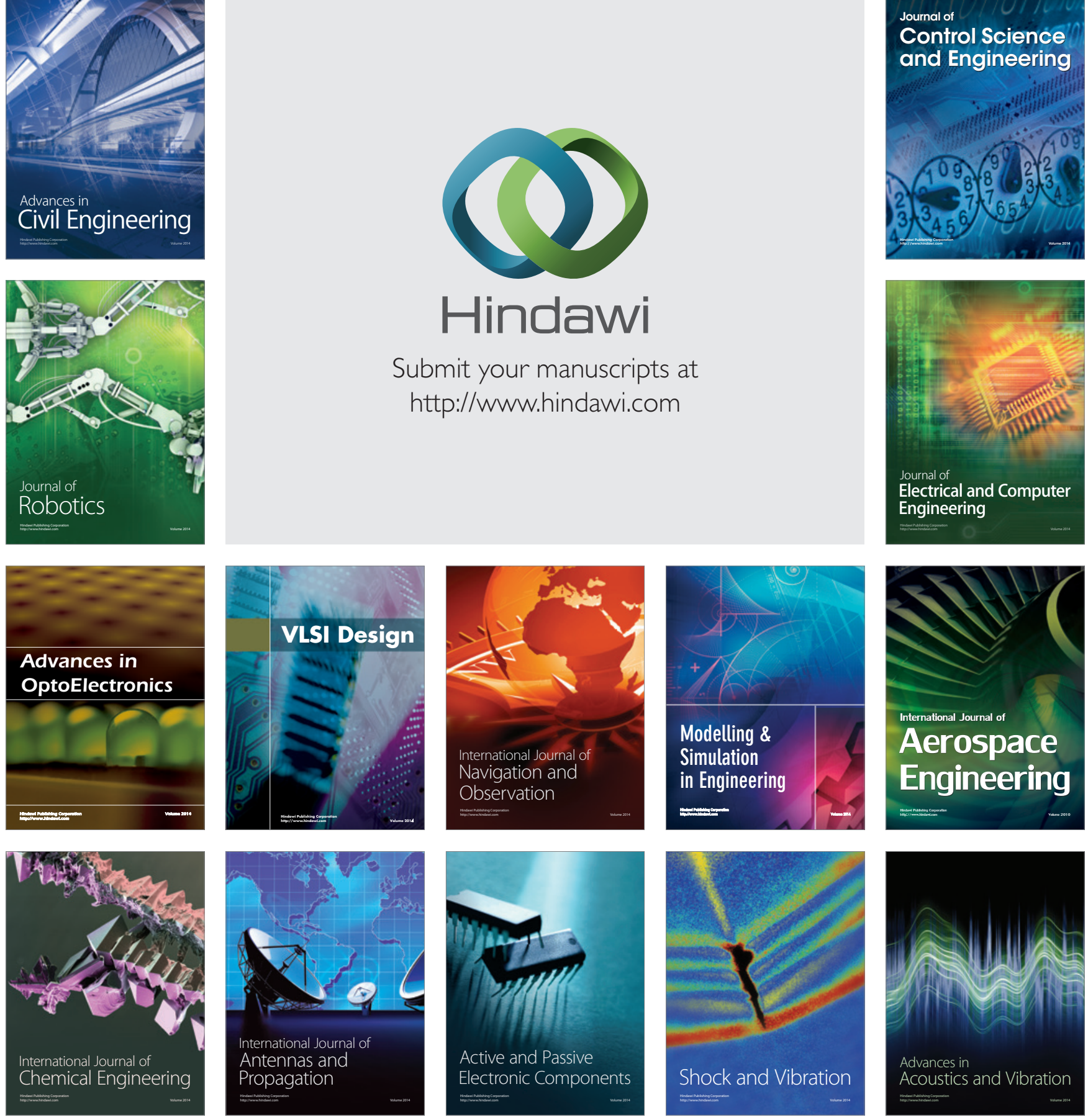